endoscopists when using MEI and VSC than without. The total CIR for all colonoscopies was greater using VSC with MEI than without (89.9\% vs $87.1 \%, p=0.0153)$. There was no significant difference in TI intubation rate, polyp detection rate or sedation used.

Conclusion Although most endoscopists prefer to use MEI and VSC when performing colonoscopy, our data suggests that the difference in caecal intubation rates with experienced endoscopists is small. However, our observed difference in CIR of $2.8 \%$ would equate to about 168 colonoscopies in our unit completed, per year that would be otherwise incomplete.

Competing interests None declared.

\section{PMO-004 DO WE NEED PROPOFOL SEDATION AND A CYTOLOGIST PRESENT DURING ENDOSCOPIC ULTRASOUND? INITIAL EXPERIENCE FROM A UK CENTRE}

\section{doi:10.1136/gutjnl-2012-302514b.4}

${ }^{1} \mathrm{~A} D$ Hopper, ${ }^{*} \mathrm{~A}$ Irvine, ${ }^{2} \mathrm{R}$ Vinayagam, ${ }^{3} \mathrm{~A}$ Dube. ${ }^{1}$ Department of Gastroenterology, Royal Hallamshire Hospital, Sheffield, UK; ${ }^{2}$ Department of Radiology, Royal Hallamshire Hospital, Sheffield, UK; ${ }^{3}$ Department of Histopathology, Royal Hallamshire Hospital, Sheffield, UK

Introduction Endoscopic ultrasound (EUS) is a prolonged procedure using endoscopes nearly twice the diameter of a standard gastroscope and relies on a compliant still patient to obtain images and fine needle aspiration (FNA) samples. Propofol or anaesthetic delivered sedation is used to ensure procedure success and tolerability in many international centres with in-room cytology expertise to optimise the yield of FNA. This practice is potentially costly and labour intensive. We retrospectively and prospectively examined tolerability, completion and FNA accuracy in a recently expanded EUS centre in the UK using midazolam and fentanyl sedation only and no in room cytologist.

Methods Electronic array radial and linear ultrasound scopes with FNA procedures were available to our centre from July 2010. A cytology processing methodology was used with complete expulsion of FNA material into a "cytorich red" medium with no in room slide processing or viewing. A standard three FNA passes was used for pancreatic masses $(20 \mathrm{ml}$ suction) and lymph nodes $(0-10 \mathrm{ml}$ suction). Accuracy was calculated with follow-up of patients for $>3$ months for specimens. Also from this time analysis of sedation used, procedure success and any reversal agent/respiratory support required was documented. From 1 September 2011 patients undergoing EUS and gastroscopy examinations were invited to complete questionnaires to score pain during the procedure.

Results From 1 July 2010 until 31 December 2011450 EUS procedures were performed. 11 were incomplete and all due to luminal stricture formation only. FNA was performed in 126 patients. Accuracy for all lesions was $84.9 \%$, and for solid pancreatic tumours $82.4 \%$ $(\mathrm{n}=68)$. Midazolam use ranged from 0 to $10 \mathrm{mg}$ (mean 3.44 median 4$)$ and fentanyl use ranged from 0 to $200 \mu \mathrm{g}$ (mean 67.9; median 50). No reversal agent was used and no patients required any assisted ventilation. Prospective recruitment from 1 September 2011 included 49 patients which participated for EUS. The mean procedure time was 19.5 min (range 8-35), mean pain score during the procedure was 2.26/10 (range 0-9 median 2), and the average dose of sedation was $3.45 \mathrm{mg}$ midazolam and $72.8 \mu \mathrm{g}$ fentanyl. During the same time period 75 consecutive patients undergoing gastroscopy participated. There was no difference in the average pain score during the procedure compared to EUS: mean $=2.6 / 10$ ( $t$ test $\mathrm{p}=0.36$ ) (mean procedure time $=6.7 \mathrm{~min}$; sedation given in $14 / 75$; mean $=3.3 \mathrm{mg}$ midazolam). Conclusion Despite prolonged procedure duration and large scope diameter, EUS procedures are safely and well tolerated with midazolam and fentanyl sedation. A high FNA accuracy can be achieved without a cytologist present in the room.

Competing interests None declared.

\section{PM0-005 BRIEF INTERVENTION REDUCES FUTURE HOSPITAL ATTENDANCE IN HAZARDOUS ALCOHOL DRINKERS}

doi:10.1136/gutjnl-2012-302514b.5

${ }^{1}$ A Assad-Sangabi, ${ }^{1} \mathrm{R}$ Smith, ${ }^{1} \mathrm{~J}$ Cleminson, ${ }^{2} \mathrm{~L}$ Jones, ${ }^{1} \mathrm{D}$ Elphick. ${ }^{1}$ Department of Gastroenterology, Chesterfield Royal Hospital, Chesterfield, UK; ${ }^{2}$ Department of Emergency Medicine, Chesterfield Royal Hospital, Chesterfield, UK

Introduction Excessive alcohol consumption is a major health burden facing the NHS. The Fast Alcohol Screening Tool (FAST) is a useful, simple tool to screen for hazardous/harmful drinking. Hazardous drinking is associated with a high risk of psychological or physical problems in the future. In this study, we assess the FAST score in patients attending the Emergency Department (ED) of our hospital. We then evaluate whether brief alcohol intervention on high scorers (hazardous drinkers), in the form of consultation and advice on alcohol related habits, results in fewer future ED attendances.

Methods All those, 18 years and over, attending the ED of Chesterfield Royal Hospital over a $24 \mathrm{~h}$ period were asked to take part in this study. All participants were formally consented. Patient demographics were taken, and reason for admission noted. Number of prior ED attendances were noted from hospital records and patient recollection. FAST questionnaires were then filled, followed by a brief intervention [typically lasting $10 \mathrm{~min}$ ] given to those who scored 3 or above. Investigators had prior training on brief intervention. Frequency of hospital attendance following this episode was then taken from hospital records.

Results 140 patients attended ED, but 25 were excluded (declined participation, life-threatening illness). Therefore, 115 patients participated in the study (60 (52\%) female and $55(48 \%)$ male; median age 47). FAST score was 0 in 60 (52\%), 1 in $13(11 \%), 2$ in $19(17 \%)$ and $\geq 3$ in $23(20 \%)$ patients. Brief alcohol intervention was carried out in 19 out of 23 patients who scored $\geq 3$ (not possible in four who were intoxicated with alcohol). These included 9 (48\%) female and 10 $(52 \%)$ male with median age of 39 . In this subgroup of patients, 15 had no previous ED attendances, one attended once, one attended twice and two attended three times each in the prior 6 months. Therefore, four out of 19 (21\%) of these patients attended ED on a total of nine occasions over that 6 months. 20 out of 92 patients $(22 \%)$ of those with FAST score $<3$ had prior admissions over the same period with a total of 25 attendances. Two out of $19(10 \%)$ patients with FAST score $\geq 3$, who were given brief intervention, reattended over the following 6 months, on one occasion each (total two attendances). 13 out of $92(14 \%)$ patients with FAST score $<3$ reattended over this time period, with total 17 attendances. Brief intervention therefore appeared to reduce future hospital attendance in hazardous alcohol drinkers.

Conclusion Hazardous alcohol drinkers (with FAST scores $\geq 3$ ) make up a large proportion of those attending ED. Brief alcohol intervention for these reduces their re-attendance. We therefore recommend front-line ED staff to be trained in brief intervention.

Competing interests None declared.

\section{PM0-006 OUT OF HOURS GASTROINTESTINAL BLEED SERVICE IN THE UK: INTER-HOSPITAL TRANSFER OF PATIENTS FOR ENDOSCOPY IS SAFE}

doi:10.1136/gutjnl-2012-302514b.6

B N Shokouhi,* M Khan, S Greenfield. Department of Gastroenterology, Queen Elizabeth II Hospital, Hertfordshire, UK

Introduction Acute Upper Gastrointestinal Bleeding (AUGIB) results in 25000 in-patient admissions annually in the UK (1). Patients admitted at weekends with AUGIB have significantly increased mortality (2). Current guidelines advise availability of out-of-hours 